Provided for non-commercial research and education use. Not for reproduction, distribution or commercial use.

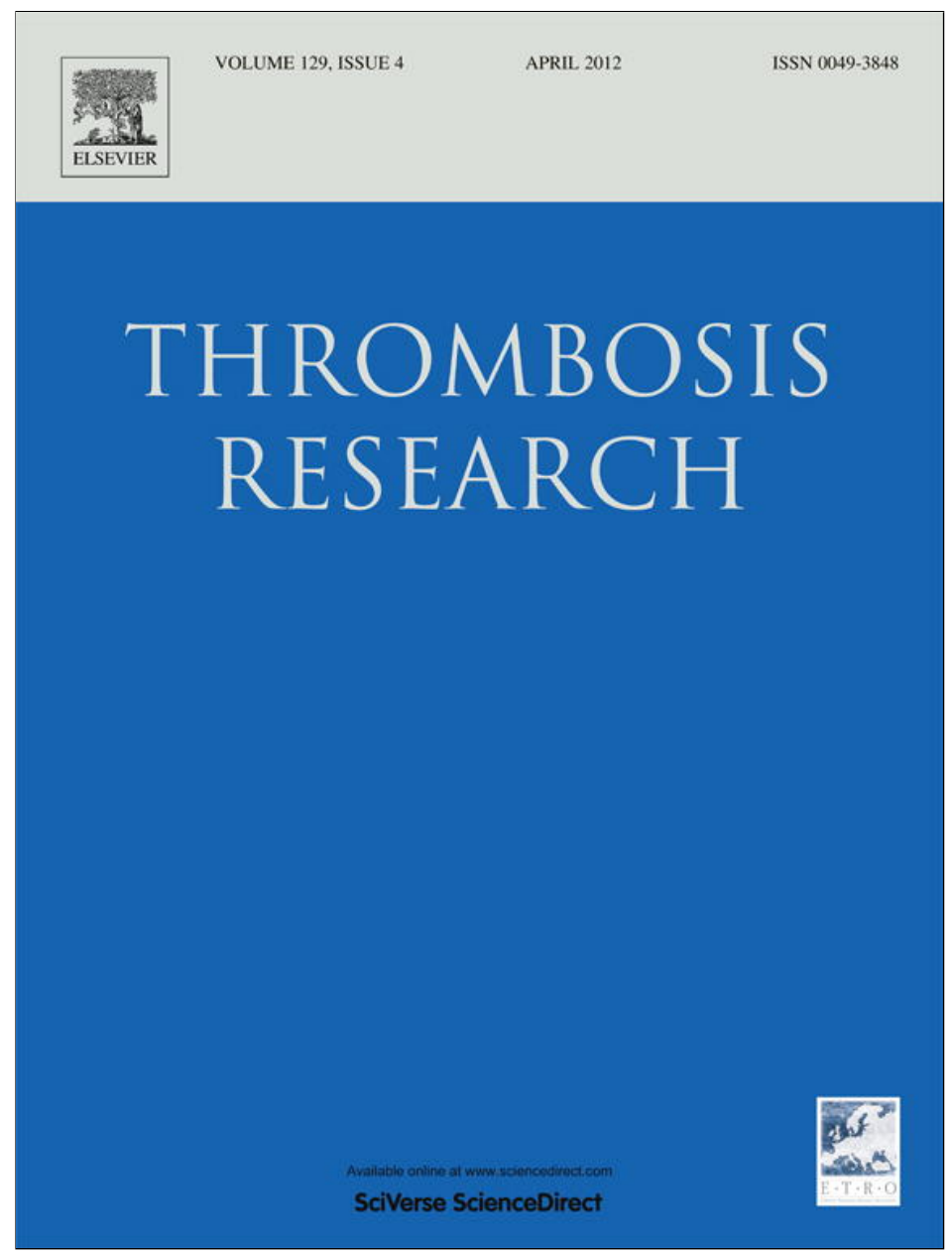

This article appeared in a journal published by Elsevier. The attached copy is furnished to the author for internal non-commercial research and education use, including for instruction at the authors institution and sharing with colleagues.

Other uses, including reproduction and distribution, or selling or licensing copies, or posting to personal, institutional or third party websites are prohibited.

In most cases authors are permitted to post their version of the article (e.g. in Word or Tex form) to their personal website or institutional repository. Authors requiring further information regarding Elsevier's archiving and manuscript policies are encouraged to visit:

http://www.elsevier.com/copyright 
Review Article

\title{
Interventional treatment of venous thromboembolism: A review
}

\author{
Davide Imberti ${ }^{a}, *$, Walter Ageno ${ }^{b}$, Roberto Manfredini ${ }^{c}$, Fabio Fabbian ${ }^{c}$, Raffaella Salmi ${ }^{d}$, \\ Rita Duce ${ }^{\mathrm{e}}$, Massimo Gallerani ${ }^{\mathrm{d}}$ \\ a Department of Internal Medicine, Piacenza Hospital, Italy \\ ${ }^{\mathrm{b}}$ Department of Internal Medicine, University of Insubria, Varese, Italy \\ c Clinica Medica, University Hospital, Ferrara, Italy \\ ${ }^{\mathrm{d}}$ Department of Internal Medicine, University Hospital, Ferrara, Italy \\ e Department of Internal Medicine, Galliera Hospital, Genova, Italy
}

\section{A R T I C L E I N F O}

\section{Article history:}

Received 7 September 2011

Received in revised form 17 October 2011

Accepted 2 November 2011

Available online 26 November 2011

\begin{abstract}
A B S T R A C T
Venous thromboembolism (VTE), including deep vein thrombosis (DVT) and pulmonary embolism (PE), is the third most common cardiovascular disease after coronary artery disease and cerebrovascular disease and is responsible for significant morbidity and mortality in the general population. Full dose anticoagulation is the standard therapy for VTE, both for the acute and the long-term phase. The latest guidelines of the American College of Chest Physicians recommend treatment with a full-dose of unfractioned heparin (UFH), lowmolecular-weight-heparin (LMWH), fondaparinux, vitamin K antagonist (VKA) or thrombolysis for most patients with objectively confirmed VTE. Catheter-guided thrombolysis and trombosuction are interventional approaches that should be used only in selected populations; interruption of the inferior vena cava (IVC) with a filter can be performed to prevent life-threatening PE in patients with VTE and contraindications to anticoagulant treatment, bleeding complications during antithrombotic treatment, or VTE recurrences despite optimal anticoagulation. In this review we summarize the currently available literature regarding interventional approaches for VTE treatment (vena cava filters, catheter-guided thrombolysis, thrombosuction) and we discuss current evidences on their efficacy and safety. Moreover, the appropriate indications for their use in daily clinical practice are reviewed.
\end{abstract}

(c) 2011 Elsevier Ltd. All rights reserved.

\section{Contents}

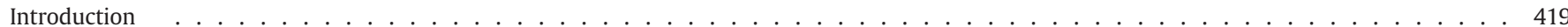

Inferior vena cava filters . . . . . . . . . . . . . . . . . . . . . . . . . . . . . . . . . . . . . . . . . . . . . 419

Permanent IVC filters . . . . . . . . . . . . . . . . . . . . . . . . . . . . . . . . . 419

Retrievable IVC filters . . . . . . . . . . . . . . . . . . . . . . . . . . . . . . . . . . . . . . . . . . . . 419

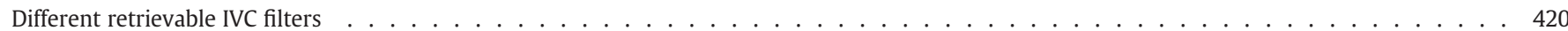

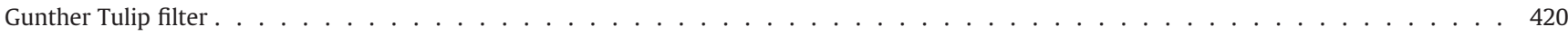

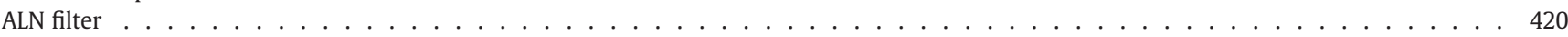

Recovery filter . . . . . . . . . . . . . . . . . . . . . . . . . . . . . . . . . . . . 420

OptEase filter . . . . . . . . . . . . . . . . . . . . . . . . . . . . . . . . . . . . 420

Indications for filter implantation . . . . . . . . . . . . . . . . . . . . . . . . . . . . . . . . . . . . 420

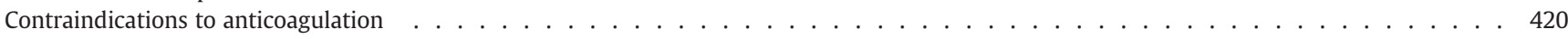

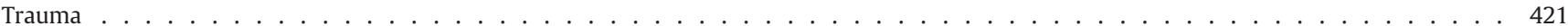

Thrombolytic therapy . . . . . . . . . . . . . . . . . . . . . . . . . . . . . . . . . . . . . . 421

Pregnancy . . . . . . . . . . . . . . . . . . . . . . . . . . . . . . . . . . . . . . 421

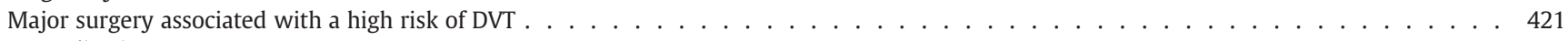

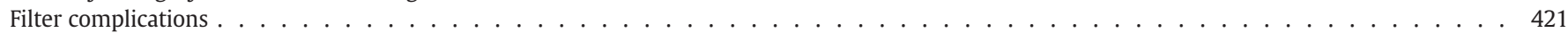

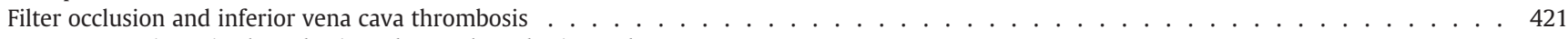

Lower extremity vein thrombosis and post-thrombotic syndrome . . . . . . . . . . . . . . . . . . . . . . . . . . . . . . . . . . . . . . 421

Vena cava perforation . . . . . . . . . . . . . . . . . . . . . . . . . . . . . . . . . 422

\footnotetext{
* Corresponding author at: Internal Medicine Department, Piacenza Hospital, Via Taverna 49, 29121 Piacenza, Italy. Tel.: + 390523302578 ; fax: + 390523 302595.

E-mail address: d.imberti@ausl.pc.it (D. Imberti).
} 


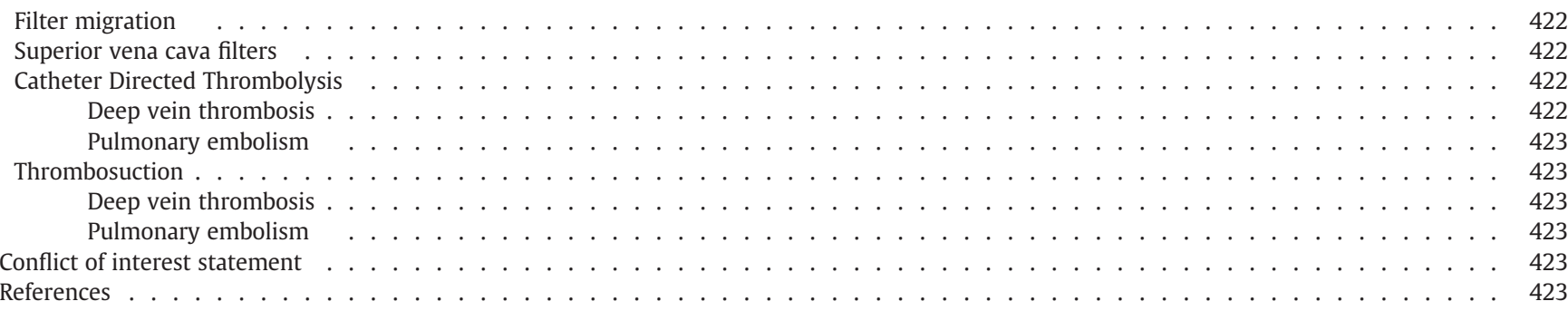

\section{Introduction}

Venous thromboembolism (VTE) consists of two related conditions: deep vein thrombosis (DVT) and pulmonary embolism (PE). The rate of first-time VTE events is approximately 100 persons/100,000 population each year in the United States, with this risk proportionally increasing with age, from less than 5 cases/100,000 persons below the age of 15 years to 500 cases/100,000 persons above the age of 80 years [1]. The basic mechanisms underlying VTE remain those previously described by Virchow: vascular endothelial damage, stasis of blood flow, and blood hypercoagulability; the most frequent risk factors include major surgery, trauma, hip fracture, lower extremity paralysis, previous VTE, increasing age, cardiac or respiratory failure, prolonged immobility, presence of central catheters, oestrogen treatment, and several inherited and acquired hematological conditions [2].

The latest guidelines of the American College of Chest Physicians [3] recommend treatment with a full-dose of unfractioned heparin (UFH), low-molecular-weight-heparin (LMWH), fondaparinux, vitamin K antagonist (VKA) or thrombolysis for most patients with objectively confirmed VTE. However, to prevent life-threatening PE in patients with VTE and contraindications to anticoagulant treatment, such as bleeding complications during antithrombotic treatment or VTE recurrences despite optimal anticoagulation, interruption of the inferior vena cava (IVC) with a filter should be sometimes considered [3-7]. Likewise, catheter-guided thrombolysis and trombosuction are interventional approaches that may be important for the management of very selected populations.

The efficacy, safety, and the appropriate indications for these therapeutic approaches are discussed herein.

\section{Inferior vena cava filters}

\section{Permanent IVC filters}

Only two randomized clinical trials have evaluated the efficacy and safety of permanent IVC filters for the prevention of PE $[8,9]$. Decousus and colleagues randomly assigned 400 patients with proximal DVT and at risk for PE to receive a vena caval filter (200 patients) or not (200 patients), in addition to standard anticoagulant therapy (LMWH, UFH, VKA) [8]. The rates of recurrent VTE, death, and major bleeding were analyzed at day 12 , and at two years. At day 12 , two patients assigned to receive filters (1.1 percent), as compared with nine patients assigned to receive no filters ( 4.8 percent), had had symptomatic or asymptomatic PE (odds ratio, $0.22 ; 95$ percent confidence interval, 0.05 to 0.90 ). At two years, 37 patients assigned to the filter group (20.8 percent), as compared with 21 patients assigned to the no-filter group (11.6 percent), had had recurrent DVT (odds ratio, 1.87; 95 percent confidence interval, 1.10 to 3.20 ). There were no significant differences in mortality or other outcomes. The authors concluded that in high-risk patients with proximal DVT the initial beneficial effect of IVC filters for the prevention of PE was counterbalanced by an excess of recurrent DVT, without any difference in mortality. The 8 years follow-up data of this population showed a rate of DVT recurrences of $34.1 \%$ in patients with filter and of $27.3 \%$ in those without $(p=0.08)$, while the incidence of post-thrombotic syndrome was surprisingly similar in the two groups (70.3\% and 69.7\%, respectively) [10].

Fullen at al enrolled in a quasi-randomised trial patients with traumatic hip fracture to receive or not a Mobin-Uddin caval filter; none of the patients received anticoagulant treatment [9]. The rate of PE was 4/41 in the filter group and 19/59 in the control group (RR $0.3,95 \%$ CI 011 to 0.82 ), showing a statistically significant reduction in the treated group; mortality was similar for filter and not-filter patients (4/41 and 14/59, respectively; RR $0.41,95 \%$ CI 0.15 to 1.16 ).

Very recently, a Cochrane review evaluated all existing published controlled clinical trials and randomised clinical trials that examined the efficacy of filters in preventing PE [11]. Only the two above mentioned studies involving a total of 529 people were included $[8,9]$ and the authors concluded that no recommendations can be drawn. On the one hand, one study showed a reduction in PE rates, but not in mortality, and it was subject to significant biases [9]. On the other hand, the PREPIC study lacked statistical power to detect a reduction in PE over shorter and more clinically significant time periods and the trial demonstrated that permanent VCFs were associated with an increased risk of long term lower limb DVT [8].

Because of the lack of strong evidences in the literature, the latest guidelines of the American College of Chest Physicians (ACCP) recommend against the routine use of a IVC filter in addition to anticoagulants in patients with DVT; the placement of an IVC filter is suggested only for patients with acute proximal DVT or PE and a contraindication to anticoagulation because of bleeding risk [3].

\section{Retrievable IVC filters}

The vast majority of the filters which have been implanted worldwide are of the permanent type [12-15]; nevertheless, placement of such filters presents a number of long term complications. Decousus and colleagues [8] demonstrated that in high-risk patients with proximal DVT the initial benefit of IVC filters for the prevention of PE was counterbalanced by an excess rate of recurrent DVT after two years of follow-up. Moreover, one of the most important long-term complications of filters is the thrombotic occlusion of the IVC, which is reported in $6 \%$ to $30 \%$ of cases $[16,17]$; other significant complications include vena cava perforation, filter dislocation, migration, rupture, fracture and fragment embolization with risk of cardiac perforation and tamponade [18]. Thus, alternative strategies for IVC interruption are required, especially in patients with a long life expectancy and for whom the need for anticoagulant therapy is presumably short.

Non-permanent filters are classified as temporary or retrievable devices. Temporary filters remain attached to a wire or catheter that exits the skin; they are often difficult to manage and present frequent complications such as thrombosis, infections or migrations [17]. They must be removed within few days of placement, which is often not enough to solve the clinical problem that had led to their placement. Retrievable filters are a new generation of IVC filters and may represent a more attractive option because they may be either left in place permanently or safely retrieved after a quite long period when they become unnecessary $[16,17]$. This optimism must be tempered by important unresolved issues, including the appropriate maximum 
implantation time, the possibility of safely and efficaciously removing the filters without being compromised by entrapped clots, and the use of anticoagulation during the implantation and peri-removal periods. A study performed in US has recently evaluated trends in the placement and removal of IVC filters in the Medicare population from 1999 through 2008 [19]. Although IVC filters were often placed not in accordance with established indications for their implantation, the frequency of their use has doubled over the past decade and the percentage of the removal was very low (ranging from $1.2 \%$ to $5.1 \%$ ) $[15,19]$. For these reasons, the FDA has recently echoed concerns about the relative infrequency with which retrievable filters are removed and has specifically recommended that "implanting physicians and clinicians responsible for the ongoing care of patients with retrievable IVC filters consider removing the filter as soon as protection from PE is no longer needed" [20].

\section{Different retrievable IVC filters}

Results of the most important studies on retrievable filters are reported in Table 1 .

\section{Gunther Tulip filter}

The Gunther Tulip filter consists of four struts of stainless wheels with hooks at the end acting as anchors. The filter can be placed either from the femoral or the jugular access, and retrieval is from the right jugular site [21]. The registry of the Canadian Interventional Radiology Association [21] and several other reports [22-26] have demonstrated the feasibility of retrieval of Gunther Tulip IVC device, after a maximum implantation time of 139 days (mean 14 days), with a low rate of recurrent PE while the filter was in place (0-3.6\%); IVC thrombosis occurred in $0-9.6 \%$ and retrieval was successful in most of the cases (ranging from $88 \%$ to $98 \%$ ).

\section{ALN filter}

The ALN filter is a hydrodinamic steel retrievable IVC filter. It has six short legs that ensure its adherence to the IVC walls, and three long legs that guarantee the correct central positioning into the vena cava [27]. ALN filter can be placed from the femoral, brachial or jugular vein approach, and can be retrieved only from the jugular approach. A number of trials have investigated the efficacy and safety of long-term retrieval of the ALN device, showing a maximum implantation time of 722 days (range 6-722 days) with a high rate of retrieval technical success (range 78\%-100\%) [27-33].

\section{Recovery filter}

The Recovery Nitinol Filter (RNF) is composed of 12 nitinol wires that extend from a nitinol sleeve and has six arms and six legs [34].

Efficacy and safety of the Recovery Filter has been evaluated in seven studies, demonstrating the feasibility of removal of the device in a high percentage of patients (retrieval technical success varying from $85 \%$ to $100 \%$ ) with a maximum implantation time of 475 days (range 5-475) [35-41].

\section{OptEase filter}

The OptEase filter is a nitinol-MRI compatible filter and it is the only filter retrievable from a femoral vein approach; the filter has a symmetrical double-basket design with six straights struts connecting the proximal and distal baskets [42].

Several trials have recently investigated the efficacy and safety of retrieval of the OptEase device, showing a maximum implantation time of 48 days (range 4-48 days) with a high percentage of removal technical success (from $85 \%$ to $100 \%$ ) [42-46].

\section{Indications for filter implantation}

\section{Contraindications to anticoagulation}

According to the 8th ACCP Evidence-Based Clinical Practice Guidelines on Antithrombotic and Thrombolytic Therapy [3,47], IVC filter placement is recommended when there is a contraindication or complication of anticoagulant therapy in a patient with proximal DVT or PE. Frequently, the contraindication to anticoagulation is temporary (i.e. haemorragic stroke, trauma) and antithrombotic therapy can be started as soon as it is resolved; for this reason, retrievable IVC filters may be the ideal "bridge" to anticoagulation for these patients. Main indications for retrievable vena cava filtration are reported in Table 2.

Table 1

Main results of retrievable filters studies.

\begin{tabular}{|c|c|c|c|c|}
\hline Study & Filter & $\begin{array}{l}\text { Number of filters } \\
\text { removed and placed }\end{array}$ & $\begin{array}{l}\text { Mean duration between filter } \\
\text { placement and retrieval (days) }\end{array}$ & $\begin{array}{l}\text { Retrieval technical } \\
\text { success }(\%)\end{array}$ \\
\hline Ponchon, 1999 [22] & Gunther-Tulip & 8 of 10 & 12; range $8-14$ & 88 \\
\hline Millward, 2001 [21] & Gunther-Tulip & 52 of 91 & 9 ; range $2-25$ & 98 \\
\hline Offner, 2003 [23] & Gunther-Tulip & 37 of 44 & 14 ; range $3-30$ & 97 \\
\hline Asch, 2002 [35] & Recovery & 24 of 32 & 53 ; range $5-134$ & 100 \\
\hline Pieri, 2003 [27] & ALN & 7 of 18 & 63; range 49-192 & 100 \\
\hline Barral, 2003 [28] & ALN & 13 of 54 & 22 ; range $11-90$ & 100 \\
\hline Pancione, 2004 [29] & ALN & 28 of 96 & 72 ; range $30-120$ & 100 \\
\hline Morris, 2004 [24] & various & 14 of 130 & $19 ;$ range $11-41$ & 93 \\
\hline Imberti, 2005 [30] & ALN & 14 of 30 & 123 ; range $30-345$ & 78 \\
\hline Grande, 2005 [36] & Recovery & 14 of 107 & 150 ; range $0-419$ & 93 \\
\hline Oliva, 2005 [39] & OptEase & 21 of 27 & $11 ;$ range $5-14$ & 100 \\
\hline Rosenthal, 2005 [43] & OptEase & 40 of 40 & 16 ; range 3-48 & 100 \\
\hline Ray, 2006 [26] & various & 80 of 197 & 19; range 1-139 & 85 \\
\hline Stefanidis, 2006 [37] & various & 47 of 83 & 142 ; range $17-475$ & 87 \\
\hline Mismetti, 2007 [31] & ALN & 56 of 220 & 51 ; range 6-352 & 93 \\
\hline Karmy, 2007 [57] & various & 90 of 446 & $28.2 \pm 26.3$ & 78 \\
\hline Pancione, 2006 [32] & ALN & 71 of 276 & 74 ; range $30-130$ & 93 \\
\hline Pellerin, 2008 [33] & ALN & 122 of 123 & 93; range 6-722 & 99 \\
\hline de Villiers, 2008 [38] & Recovery & 22 of 54 & 48 ; range 7-90 & 96 \\
\hline Oliva, 2008 [39] & Recovery & 51 of 120 & 53 ; range $7-242$ & 100 \\
\hline Binkert 2009 [41] & Recovery & 61 of 100 & 140 ; range $5-300$ & 95 \\
\hline Onat, 2009 [45] & OptEase & 124 of 228 & 11 ; range $4-23$ & 91 \\
\hline Kalva, 2011 [46] & OptEase & 14 of 71 & 9 ; range $5-21$ & 85 \\
\hline
\end{tabular}


Table 2

Indications for retrievable IVC filters implantation.

\author{
Appropriate indication \\ Temporary contraindication to anticoagulation \\ Potential indications \\ Prophylaxis in high risk trauma patients \\ Thrombolysis of ilio-caval thrombus \\ Pregnancy \\ Prophylaxis in high risk major orthopedic surgery \\ Prophylaxis in bariatric surgery
}

\section{Trauma}

Thromboprophylaxis is an important issue in patients with major trauma [48], and PE is the cause of death in $20 \%$ of severely injured patients [49]. Management of thromboprophylaxis may be problematic because of the limited efficacy of standard prevention (low dose heparin, sequential compression devices) and concern about potential bleeding complications associated with anticoagulant treatment. For these reasons, in the last few years an increasing interest in the use of IVC filter for PE prophylaxis in this clinical setting has been observed [47]. Unfortunately, there are no randomized trials demonstrating a clear benefit of IVC insertion in trauma patients [48-57]. Three small studies have reported a low rate of PE in patients with severe polytrauma who underwent prophylactic IVC filter insertion [54-56]. In a large prospective study 127 multitrauma patients underwent a prophylactic placement of a retrievable IVC filters (Gunther Tulip $n=49$; Recovery $n=41$; OpTease $n=37$ ), without any complication [53]. Sixty-six patients underwent uneventful retrieval of IVC filters after 5-116 days from implantation (mean 71 days), while in 45 retrieval was not attempted (41 due to contraindication to anticoagulation and 4 because of trapped emboli within the filter). Finally, a retrospective review of 446 trauma patients receiving retrievable IVC filters in 21 different participating centers was performed (Gunther Tulip $n=152$; Recovery $n=224$; OpTease $n=37$ ) [57]. Of interest, only $22 \%$ of the implanted filters were retrieved; the main reason for which IVC filters were not removed was because of loss to follow-up (31\%). Of 115 patients in whom retrieval was attempted, removal failed for technical reasons in 15 patients and because of significant residual thrombus within the filter in 10 patients.

\section{Thrombolytic therapy}

Systemic thrombolysis of proximal DVT and IVC thrombi has resulted in several cases of fatal and non fatal PE; therefore, prophylactic placement of IVC filters has been proposed as a strategy to prevent PE in patients undergoing thrombolysis. A European multicenter registry of temporary IVC filters used during systemic thrombolysis showed an incidence of fatal PE of $2.1 \%$ and of non fatal PE of $1.6 \%$ [58]. To our knowledge, no data have been published regarding retrievable IVC filters during thrombolysis for DVT; because of their ease of use and of their advantages, these devices appear as potentially attractive alternatives to temporary filters. On the other hand, thrombolysis is in principle contraindicated as a first approach to DVT treatment [3], thus the potential use of filters in this setting would be extremely limited.

\section{Pregnancy}

The overall incidence of VTE complications during pregnancy ranges from $0.2 \%$ to $1.2 \%$, and is even higher during the puerperium [59]. Since pregnancy is typically a temporary risk factor for VTE, the use of a non-permanent filter is particularly appealing when anticoagulation is contraindicated. In a multicenter study, about $3 \%$ of all temporary filters inserted were placed in pregnant women who were undergoing caesarean section and thrombectomy [60].
Few case reports showed that retrievable IVC filters offer a safe and effective prevention to PE during pregnancy and puerperium and can be removed without complications $[61,62]$. On the other hand, there are no strong data supporting the routine use of IVC filters in patients suffering from acute DVT during pregnancy and this device should be reserved for selected and specific situations.

\section{Major surgery associated with a high risk of DVT}

Patients undergoing major orthopaedic surgery such as hip and knee replacement carry a very high risk of VTE complications [47]. Several case series showed the efficacy of IVC filters in the prevention of PE in orthopaedic patients, but none of these studies included a control group and follow-up was of limited intensity and duration [29]. Furthermore many recent advances in pharmacological prophylaxis (low-molecular weight heparin, synthetic factor Xa and thrombin inhibitors) have contributed to significantly reduce the risk of VTE in this setting. Retrievable filters remain a useful option for highly selected cases, i.e. patients at very high thromboembolic risk because of a previous, recent massive PE or recurrent VTE episodes or patients with a major contraindication to pharmacologic therapy [30].

$\mathrm{PE}$ is considered the leading cause of death after bariatric surgery and common pharmacologic prophylactic strategies have not been adequately tested in morbidly obese patients $[50,63]$. Placement of IVC filters has become a common prophylactic strategy among some bariatric surgeons, even if no prospective randomized clinical trials have compared IVC filters with alternative methods. However, filter placement can be challenging in these patients, especially in the super obese (BMI>60). In conclusion, there are no data supporting the routine use of retrievable IVC filters in bariatric patients, and this device should be reserved for specific situations.

\section{Filter complications}

The most important filter complications are reported in Table 3.

\section{Filter occlusion and inferior vena cava thrombosis}

Occlusion of the filter is the most frequent complication of vena cava filters and its incidence varies from $6 \%$ to $30 \%$ of cases [16-19]. The reasons for this complication include thrombogenicity of the device, natural cephalic progression of DVT from the lower limb and entrapment of emboli within the filter. Thrombosis of the filter and vena cava occlusion may be associated with important clinical side effects, including decreased protection against PE, migration of the filter, post-thrombotic syndrome and chronic venous stasis. New generation filters offer the advantage of a lower thrombogenicity compared with older ones.

\section{Lower extremity vein thrombosis and post-thrombotic syndrome}

Vena cava filters themselves have sometimes been observed to obstruct blood flow and contribute to an increase of recurrence of DVT of the lower extremity $[8,10,64]$. For these reason, the ACCP guidelines recommend, if the filter is positioned as an alternative to

Table 3

Main complications of inferior vena cava filters.

\begin{tabular}{ll}
\hline Complication & Rate (\%) \\
\hline Complications from insertion & $4-11$ \\
Insertion site thrombosis & $2-28$ \\
IVC thrombosis & $6-30$ \\
Filter migration & $3-69$ \\
IVC perforation & $9-24$ \\
Post-thrombotic syndrome & $5-70$ \\
\hline
\end{tabular}


anticoagulation, beginning of adequate anticoagulant therapy as soon as possible if the risk of bleeding resolves [3]. Otherwise, the optimal duration of anticoagulation in patients with permanent or optional filter that is left in situ is still uncertain. A recently published cohort study followed patients who had VTE, followed by treatment with permanent IVC filter placement and were anticoagulated long-term as soon as safety allowed [64]. Patients underwent annual physical examinations and ultrasound surveillance of the lower extremity deep veins and of the IVC filter site. Symptomatic DVT occurred in 24 of 121 patients (20\%; 95\% CI, 14\%-28\%); symptomatic PE (one fatal) was diagnosed in six patients (5\%; $95 \% \mathrm{CI}, 2 \%-10 \%)$. There were 45 episodes of filter clot in 36 patients (30\%; 95\% CI, 22\%-38\%). The rate of major bleeding (6.6\%) was similar to that of a concurrent persistently anticoagulated cohort without IVC filters (5.8\%). Thus, the authors suggest indefinite anticoagulation to IVC filter recipients if contraindications to anticoagulation remit. On the contrary, other data of the literature, although limited, do not seem to show significant differences in the risk of DVT recurrences after IVC filter placement with or without anticoagulation [65]. To sum up, in absence of strong evidence in the literature and waiting for the results of well-designed clinical trials, patients with IVC filter should receive anticoagulation therapy according to current guidelines in any specific clinical situation; it is not suggested to continue indefinite anticoagulation just because the filter is still present.

Finally, the association of vena cava filters with an increase of post-thrombotic syndrome is still matter of debate; the available data suggest the potential risk of post-thrombotic syndrome during long-term follow-up in patients with permanent IVC filters $[8,10]$.

\section{Vena cava perforation}

Vena cava perforation is a usually asymptomatic complication, and without substantial clinical importance. Frequently, it is only a radiological finding which occurs when filter components extend more than $3 \mathrm{~mm}$ outside of the wall of the IVC [64]. More rarely, bleeding complications are associated with vena cava perforation, usually when the filter leg is withdrawn leaving an open hole; other severe consequences have been rarely reported [66,67].

\section{Filter migration}

The migration of the filter towards the heart is a potentially lifethreatening complication of IVC filters, even if, in the majority of cases, migration is minor and does not result in any significant morbidity [30]. A multicenter registry found that temporary IVC filters had a dislocation rate of $4.8 \%$; no death due to this complication was reported [58]. A recently published paper reported a high rate of strut fracture (16\%) and fragments embolization (25\%) of the Bard retrievable IVC filter; of interest, three out of 28 patients experienced life-threatening cardiac complications related to migration of fragments to the heart [18].

\section{Superior vena cava filters}

The placement of superior vena cava (SVC) filters to prevent PE from upper-extremity DVT (UEDVT), although controversial, has been reported. A recently published review identified a total of 21 publications that included 209 SVC filters and documented eight major filterrelated complications (3.8\%), including four cardiac tamponades, two aortic perforations, and one recurrent pneumothorax [68]. The inhospital or 1 -month mortality rate was $43.1 \%$. Twenty-eight additional publications were identified which reported 3,747 cases of UEDVT. The rates of PE and associated mortality were $5.6 \%$ and $0.7 \%$, respectively. Studies imaging both upper and lower extremities found DVT 14.7 times more likely to occur in the lower extremities than in the upper extremities and the rate of PE from a lower-extremity thrombus to be
$25.1 \%$. The lack of evidence documenting the risk from UEDVT and the absence of data supporting the safety and efficacy of SVC filters bring their benefit into question.

\section{Catheter Directed Thrombolysis}

\section{Deep vein thrombosis}

Treatment of lower limb DVT should be started as soon as possible to maximally reduce the risk of further complications such as PE, recurrent DVT or post-thrombotic syndrome (PTS) [69]. PTS occurs in 20 to $50 \%$ of patients after acute DVT and leg ulceration is present in up to $10 \%$ of patients $[70,71]$. These conditions lead to disability and reduced quality of life, with important clinical and public health implications, as more than $50 \%$ of patients are of working age. Oral anticoagulant therapy reduces thrombus propagation, but does not effectively produce clot lysis, thus potentially resulting in an incomplete prevention of PTS, which occurs after proximal DVT in up to $50 \%$ of patients within two years [70,71]. Treatments that actively remove the clots have the potential to reduce acute symptoms and the risk of PTS by directly reversing venous obstruction and restoring the function in valves that were immobilized by the thrombus.

The effectiveness of systemic thrombolysis to achieve early clot lysis had been investigated in a number of trials which found it to be associated with high rates of serious bleeding complications with relatively modest rates of thrombus clearance [72-74]. Catheterdirected thrombolysis (CDT) involves delivery of thrombolytic agents directly through a catheter traversing the thrombus. This may be more effective in achieving local clot lysis and in restoring venous patency while significantly reducing the risk of systemic bleeding complications.

Most of the studies on catheter-directed thrombolysis are observational studies or case-series [75-83]. The long-term outcome of catheter-directed thrombolysis in patients with acute iliofemoral venous thrombosis was evaluated in 101 patients with 103 extremities affected by iliofemoral venous thrombosis [84]. At 6 years, $82 \%$ of the limbs treated with CDT had patent veins with competent valves and without any skin changes or venous claudication. In the National Venous Registry, patients with short-term thrombosis ( $<10$ days) had better outcomes than those with older clots and correction of underlying venous lesions after successful thrombolysis, usually with intravascular stenting, appeared to be beneficial [76]. In an evaluation of 98 patients with iliofemoral DVT treated with CDT $(n=68)$ or anticoagulation $(n=30)$, quality of life was better in patients treated with CDT and correlated with the degree of lysis [81].

Few trials compared catheter-directed thrombolysis with conventional anticoagulant treatment. A single-center trial [85] randomly selected 35 patients with acute iliofemoral DVT to catheter-directed intrathrombus streptokinase or to anticoagulation alone. Six-months after treatment, patency rate was significantly higher in the thrombolysis group ( $72 \%$ vs $12 \%, \mathrm{p}<0.001$ ), and the prevalence of venous reflux was lower. In a subsequent, multicenter, controlled trial, 103 patients were allocated to additional $\operatorname{CDT}(n=50)$ or to standard treatment alone $(n=53)$ [86]. After CDT, complete lysis was achieved in 24 and partial (50\%-90\%) lysis in 20 patients. After 6 months, iliofemoral patency was found in $32(64.0 \%)$ in the CDT group vs. 19 (35.8\%) controls, corresponding to an absolute risk reduction (RR) of $28.2 \%$. Although bleeding complications are the major concern with lytic therapy, recent reports have shown bleeding complication rates to be less than half the rates in earlier reports, which is likely due to more appropriate patient selection and experience with the technique. Data are not available for the comparison between different plasminogen activators or between a particular catheter or catheter-based technique to others, and there are insufficient data to assess the additional benefits of IVC filters in this setting. Overall, the results of published studies suggest that CDT may be effective in selected patients; however, indications for CDT are mainly based on 
the results of few and small RCTs. Thus, the latest ACCP guidelines suggest CDT only in selected patients with extensive acute proximal DVT (iliofemoral DVT, symptoms for $<14$ days, good functional status, life expectancy $>1$ year) who have a low risk of bleeding, if appropriate expertise and resources are available [3].

\section{Pulmonary embolism}

It has been postulated that direct, intra-embolic infusion of thrombolytics into large proximal emboli might be more beneficial than the peripheral route. In patients with acute PE, thrombolytic therapy administered directly into the pulmonary arteries does not appear to be more beneficial than that given by peripheral administration, and infusion of rt-PA directly into a pulmonary artery as opposed to a peripheral vein does not accelerate thrombolysis, but causes more frequent bleeding at the catheter insertion site. In fact, the results from a pilot trial indicate that intrapulmonary infusion of rt-PA does not offer significant benefit over the intravenous route [87]. When a lytic agent is appropriate for PE, current evidence supports that thrombolytic therapy should be infused into a peripheral vein over 2 hours or less; in particular, rt-PA administered at a dose of $100 \mathrm{mg}$ over 2 hours, is currently the most widely used and evaluated regimen. Based on the available literature, the last guidelines of the ACCP recommend that in patients with acute PE, thrombolytic treatment should be administered via a peripheral vein rather than placing a pulmonary artery catheter [3].

\section{Thrombosuction}

\section{Deep vein thrombosis}

The combination of mechanical thrombus fragmentation (associated or not with suction) and catheter-directed thrombolysis (CDT) is frequently used in the daily clinical practice of centers performing CDT (pharmacomechanical thrombolysis). Although randomized clinical trials comparing CDT and pharmacomechanical thrombolysis are not available, retrospective data $[88,89]$ show that the two procedures have similar efficacy and safety based on the rates of successful thrombolysis and of major bleeding, respectively. However, pharmacomechanical thrombolysis is associated with shorter treatment times, fewer days of hospitalization and is less expensive.

Based on the available literature, the latest guidelines of the ACCP suggest in patients with acute DVT the use of pharmacomechanical thrombolysis (including thrombus fragmentation and/or suction) in preference to CDT alone, with the aim to shorten treatment time if appropriate expertise and resources are available [3]. Actually, large and well-designed randomized clinical trials assessing the role of CDT and mechanical thrombectomy for reducing the incidence of postthrombotic syndrome in patients with DVT are still ongoing (ATTRACT, CaVenT and DUTCH CAVA trials). However, waiting the results of these studies, pharmacomechanical thrombolyis should be used only in appropriate patients who do not have any contraindications to thrombolysis (Table 4).

\section{Pulmonary embolism}

Hemodynamically unstable PE, defined as PE with arterial hypotension or cardiogenic shock at presentation, is associated with a poor short term prognosis. Systemic thrombolysis is the mainstay of therapy in this setting, but its use remains associated with an estimated $20 \%$ risk of major hemorrhage, including a 3\%-5\% risk of hemorrhagic stroke. Although rarely performed, surgical embolectomy is a potential alternative treatment. However, the presence of contraindications to fibrinolytic therapy are rather common in the setting of high-risk PE, and surgical embolectomy is frequently not performed owing to excessively high surgical risk. Under those circumstances, interventional catheterization techniques (including percutaneous, catheter-based thrombectomy, thrombus fragmentation, clot pulverization, rheolytic thrombectomy, and rotational catheter embolectomy) [90-94] are
Table 4

Contraindications to thrombolytic therapy.

\author{
Absolute contraindications \\ Haemorrhagic stroke or stroke of unknown origin at any time \\ Ischaemic stroke in preceding 6 months \\ Central nervous system damage or neoplasms \\ Recent major trauma/surgery/head injury (within preceding 3 weeks) \\ Gastrointestinal bleeding within the last month \\ Known bleeding \\ Relative contraindications \\ Transient ischaemic attack in preceding 6 months \\ Oral anticoagulant therapy \\ Pregnancy or within 1 week post partum \\ Non-compressible punctures \\ Traumatic resuscitation \\ Refractory hypertension (systolic blood pressure $>180 \mathrm{mmHg}$ ) \\ Advanced liver disease \\ Infective endocarditis \\ Active peptic ulcer
}

reasonable alternatives for hemodynamically unstable PE. Pharmacologic thrombolysis and mechanical interventions can also be done together in absence of high bleeding risk.

There are no randomized clinical trials or prospective well conducted cohort studies that have evaluated the efficacy and safety of interventional catheterization techniques in this clinical setting. Several case series [90-93] enrolling a small number of patients suggest that these techniques can be lifesaving in very selected clinical situations. A recently published meta-analysis evaluated the safety and efficacy of modern catheter-directed treatment (CDT) for the therapy of massive PE [95]. Modern techniques were defined, as the use of lowprofile devices ( $<$ or $=10 \mathrm{~F}$ ), mechanical fragmentation and/or aspiration of emboli including rheolytic thrombectomy, and intraclot thrombolytic injection if a local drug was infused. Clinical success was defined as stabilization of hemodynamics, resolution of hypoxia, and survival to hospital discharge. Five hundred and ninety-four patients from 35 studies (six prospective, 29 retrospective) met the criteria for inclusion. For mechanical intervention, the most common technique used was rotating pigtail fragmentation of emboli, performed in $69 \%$ of the patients (408/594). The pooled clinical success rate from CDT was $86.5 \%$ (95\% confidence interval [CI]: $82.1 \%, 90.2 \%$ ). Pooled risks of minor and major procedural complications were 7.9\% (95\% CI: 5.0\%, $11.3 \%$ ) and $2.4 \%$ (95\% CI: $1.9 \%, 4.3 \%$ ), respectively. Data on the use of systemic thrombolysis before CDT were available in 571 patients; 546 of those patients (95\%) were treated with CDT as a first adjunct to heparin without previous intravenous thrombolyis. The latest guidelines of the ACCP recommend that interventional catheterization techniques should not be used for most patients with PE; this approach is reasonable only if appropriate expertise is available in selected highly compromised patients who are unable to receive thrombolytic therapy because of bleeding risk, or whose critical status does not allow sufficient time for systemic thrombolytic therapy to be effective [3].

\section{Conflict of interest statement}

All authors have no direct or indirect conflicts of interest to disclose.

\section{References}

[1] White RH. The epidemiology of venous thromboembolism. Circulation 2003;107: I-4-I-8.

[2] Anderson Jr FA, Spencer FA. Risk factors for venous thromboembolism. Circulation 2003;107:I-9-I-16.

[3] Kearon C, Kahn SR, Agnelli G, et al. American College of Chest Physicians. Antithrombotic therapy for venous thromboembolic disease: American College of Chest Physicians Evidence-Based Clinical Practice Guidelines (8th Edition). Chest 2008 Jun;133(6 Suppl):454S-545S.

[4] Tovey C, Wyatt S. Diagnosis, investigation and management of deep vein thrombosis. BMJ 2003;326:1180-4.

[5] Mobin-Uddin K, Smith PE, Martinez LD, et al. A vena cava filter for the prevention of pulmonary embolism. Surg Forum 1967;18:209-11. 
[6] Cimochowski GE, Evans RH, Zarins CK, et al. Greenfield filter versus Mobin-Uddin umbrella: the continuing quest for the ideal method of vena cava interruption. J Thorac Cardiovasc Surg 1980;79:358-65.

[7] Greenfield LJ, McCurdy JR, Brown PP, et al. A new vena cava filter permitting continued flow and resolution of emboli. Surgery 1973;73:599-606.

[8] Decousus H, Leizorovicz A, Parent F, et al. A clinical trial of vena cava filters in the prevention of pulmonary embolism in patients with proximal deep-vein thrombosis. N Engl J Med 1998;338:409-15.

[9] Fullen WD, Miller EH, Steele WF, McDonough JJ. Prophylactic vena cava interruption in hip fractures. J Trauma 1973;13:403-10.

[10] The PREPIC Study Group. Eight-year follow-up of patients with permanent vena cava filters in the prevention of pulmonary embolism. Circulation 2005;112:416-22.

[11] Young T, Tang H, Hughes R. Vena caval filters for the prevention of pulmonary embolism. Cochrane Database Syst Rev 2010 Feb 17;2:CD006212.

[12] Athanasoulis C. Inferior vena cava filters: review of a 26 -year single center clinical experience. Radiology 2000;216:54-66.

[13] Stein PD, Kayali F, Olson RE. Twenty-one year trends in the use of inferior vena cava filters. Arch Intern Med 2004;164:1541-5.

[14] Hull RD. Changes in the technology of inferior vena cava filters promise benefits to the patient with less harm, but a paucity of evidence exists. J Thromb Haemost 2005;3:1368-9.

[15] Spencer FA, Bates SM, Goldberg RJ, et al. A population-based study of inferior vena cava filters in patients with acute venous thromboembolism. Arch Intern Med 2010:170:1456-62.

[16] Millward SF, Grassi CJ, Kinney TB, et al. Technology Assessment Committee of the Society of Interventional Radiology. Reporting standards for inferior vena caval filter placement and patient follow-up: supplement for temporary and retrievable/optional filters. J Vasc Interv Radiol 2005;16:441-3.

[17] Imberti D, Ageno W, Carpenedo M. Retrievable vena cava filters: a review. Curr Opin Hematol 2006;13:351-6.

[18] Nicholson W, Nicholson WJ, Tolerico P, et al. Prevalence of fracture and fragment embolization of Bard retrievable vena cava filters and clinical implications including cardiac perforation and tamponade. Arch Intern Med 2010;170:1827-31.

[19] Duszak R, Parker L, Levin D, et al. Placement and removal of inferior vena cava filters: national trends in the Medicare population. J Am Coll Radiol 2011;89:483-9.

[20] U.S. Food and Drug Administration. Removing retrievable inferior vena cava filters: initial communication. Available at:http://www.fda.gov/MedicalDevices/Safety/ AlertsandNotices/ucm221676.htm Accessed November 1, 2010.

[21] Millward SF, Oliva VL, Bell SD, et al. Gunther-Tulip retrievable vena cava filter: results from the registry of the Canadian Interventional Radiology Association. J Vasc Interv Radiol 2001;12:1053-8.

[22] Ponchon M. Temporary vena cava filtration. Preliminary experience with removable vena cava filters. Acta Clin Belg 1999;54:223-8.

[23] Offner PJ, Hawkes A, Madayag R, et al. The role of temporary inferior vena cava filters in critically ill surgical patients. Arch Surg 2003;138:591-5.

[24] Morris CS, Rogers FB, Najarian KE, et al. Current trends in vena cava filtration with the introduction of a retrievable filter at a level I trauma center. I Traum 2004;57:32-6.

[25] Neuerburg GM, Gunther RW, Vorwerk D, et al. Results of a multicenter study of the retrievable Tulip vena cava filters: preliminary early clinical experience. Cardiovasc Intervent Radiol 1997;20:10-6.

[26] Ray CE, Mitchell E, Zipser S, et al. Outcomes with retrievable inferior vena cava filters: a multicenter study. J Vasc Interv Radiol 2006;17:1595-604.

[27] Pieri S, Agresti M, Morucci M, et al. Optional vena cava filters: preliminary experience with a new vena cava filter. Radiol Med 2003;105:56-62.

[28] Barral F, Tardy B, Guillot K, et al. Clinical experience with optional cava filters. J Thromb Haemost 2003;1(Suppl 1):OC441.

[29] Pancione L, Mecozzi B. Permanent/removable vena cava filter ALN (France): our experience with 96 patients. Abstract SSJ03, Proceedings of 90th Annual Meeting of the Radiological Society of North America, Chicago, 28 November-3; 2004 December.

[30] Imberti D, Bianchi M, Farina A, et al. Clinical experience with retrievable vena cava filters: results of a prospective observational study. J Thromb Haemost 2005;3: 1370-5.

[31] Mismetti P, Rivron-Guillot K, Quenet S, et al. A prospective long-term study of 220 patients with a retrievable vena cava filter for secondary prevention of venous thromboembolism. Chest 2007;131:223-9.

[32] Pancione L, Pieri S, Agresti P, et al. Use of the ALN permanent/removable vena cava filter. A multi-centre experience Minerva Chir 2006;61:501-7.

[33] Pellerin O, Barral FG, Lions C, et al. Early and late retrieval of the ALN removable vena cava filter: results from a multicenter study. Cardiovasc Intervent Radiol 2008;31:889-96

[34] Rosenthal D, Wellons ED, Lai KM, Bikk A, Henderson VJ. Retrievable inferior vena cava filters: initial clinical results. Ann Vasc Surg 2006;20:157-65.

[35] Asch MR. Initial experience in humans with a new retrievable inferior vena cava filter. Radiology 2002;225:835-44.

[36] Grande WJ, Trerotola SO, Reilly PM, et al. Experience with the Recovery filter as a retrievable inferior vena cava filter. J Vasc Interv Radiol 2005;16:1189-93.

[37] Stefanidis D, Paton BL, Jacobs DG, et al. Extended interval for retrieval of vena cava filters is safe and may maximize protection against pulmonary embolism. Am J Surg 2006;192:789-94.

[38] de Villiers L, Mackenzie S, Gibbs H, et al. Initial Australian experience with the recovery inferior vena cava filter in patients with increased risk of thromboembolic disease. J Med Imaging Radiat Oncol 2008;52:124-9.

[39] Oliva VL, Perreault P, Giroux MF, et al. Recovery G2 inferior vena cava filter: technical success and safety of retrieval. J Vasc Interv Radiol 2008;19:884-9.
[40] Cantwell CP, Pennypacker J, Singh H, et al. Comparison of the recovery and G2 filter as retrievable inferior vena cava filters. J Vasc Interv Radiol 2009;20:1193-9.

[41] Binkert CA, Drooz AT, Caridi JG, et al. Technical success and safety of retrieval of the G2 filter in a prospective, multicenter study. J Vasc Interv Radiol 2009;20: 1449-53.

[42] Oliva VL, Szatmari F, Giroux MF, et al. The Jonas study: evaluation of the retrievability of the Cordis OptEase inferior vena cava filter. J Vasc Interv Radiol 2005;16:1439-45

[43] Rosenthal D, Swischuk JL, Cohen SA, Wellons ED. OptEase Retrievable Inferior Vena Cava Filter: Initial Multicenter Experience. Vascular 2005;13:286-9.

[44] Ziegler JW, Dietrich GJ, Cohen SA, et al. PROOF trial: protection from pulmonary embolism with the OptEase filter. J Vasc Interv Radiol 2008;19:1165-70.

[45] Onat L, Ganiyusufoglu AK, Mutlu A, et al. OptEase and TrapEase vena cava filters: a single-center experience in 258 patients. Cardiovasc Intervent Radiol 2009;32: 992-7.

[46] Kalva SP, Marentis TC, Yeddula K, et al. Long-term safety and effectiveness of the "OptEase" vena cava filter. Cardiovasc Intervent Radiol 2011;34:331-7.

[47] Geerts WH, Bergqvist D, Pineo GF, et al. Prevention of venous thromboembolism. American College of Chest Physicians. Antithrombotic therapy for venous thromboembolic disease: American College of Chest Physicians Evidence-Based Clinical Practice Guidelines (8th Edition). Chest 2008 Jun;133(6 Suppl):381S-453S.

[48] Geerts WH, Code JI, Jay RM, et al. A prospective study of venous thromboembolism after major trauma. N Engl J Med 1994;331:1601-6.

[49] Sevitt S. Fatal road accidents: injuries, complications, and causes of death in 250 subjects. Br J Surg 1968;55:481-505.

[50] Rutherford RB. Prophylactic indications for vena cava filters: critical appraisal. Semin Vasc Surg 2005;18:158-65.

[51] Imberti D, Squizzato A, Ageno W. A survey of thromboprophylaxis management in patients with major trauma. J Pathophysiol Haemost Thromb 2005;34:249-54.

[52] Rectenwald JE. Vena cava filters: uses and abuses. Semin Vasc Surg 2005;18: 166-75.

[53] Gosin IS, Graham AM, Ciocca RG, et al. Efficacy of prophylactic vena cava filters in high risk trauma patients. Ann Vasc Surg 1997;11:100-5.

[54] Rogers FB, Shackford SR, Ricci MA, et al. Routine prophylactic vena cava filter insertion in severely injured patients decreases the incidence of pulmonary embolism. J Am Coll Surg 1995;180:641-7.

[55] Spain DA, Richardson JD, Polk HC, et al. Venous thromboembolism in the high-risk trauma patient: do risks justify aggressive screening and prophylaxis ? J Trauma 1997:42:463-7.

[56] Rogers FB. Venous thromboembolism in trauma patients: a review. Surgery $2001 ; 130: 1-12$

[57] Karmy-Jones R, Jurkovich G, Velmahos G, et al. Practice patterns and outcomes of retrievable vena cava filters in trauma patients: an AAST multicenter study. J Trauma 2007;62:17-25.

[58] Lorch H, Welger D, Wagner V, et al. Current practice of temporary vena cava filter insertion: a multicenter registry. J Vasc Interv Radiol 2000;11:83-8.

[59] Bates SM, Greer IA, Pabinger I, et al. Venous thromboembolism, thrombophilia, antithrombotic therapy and pregnancy. American College of Chest Physicians Evidence-Based Clinical Practice Guidelines (8th Edition). Chest 2008 Jun;133(6 Suppl):844S-86S

[60] Neil AM, Appleton DS, Richards P. Retrievable inferior vena cava filters for thromboembolic disease in pregnancy. Br J Obstet Gynaecol 1997;104:1416-7.

[61] Neri E, Civeli L, Benvenuti A, et al. Protected ileofemoral venous thrombectomy in a pregnant woman with pulmonary embolism and ischemic vein thrombosis. Tex Heart Int J 2002;29:130-2.

[62] Ishikawa T, Okabe T, Ogawa K, et al. Usefulness of a second temporary vena cava filter for preventing acute pulmonary thromboemblism. Circ J 2003;67:718-20.

[63] Rajasekhar A, Crowther M. Inferior vena caval filter insertion prior to bariatric surgery: a systematic review of the literature. J Thromb Haemost 2010;8: 1266-70.

[64] Hajduk B, Tomkowski WZ, Malek G. Davidson BL Vena cava filter occlusion and venous thromboembolism risk in persistently anticoagulated patients: a prospective, observational cohort study. Chest 2010;137:877-82.

[65] Pons M, Riglietti A, Van den Berg JC. The role of vena cava filters in the management of venous thromboembolism. J Cardiovasc Surg 2010;51:355-64.

[66] Failla PJ, Reed KD, Summer WR, Karam GH. Inferior vena caval filters: key considerations. Am J Med Sci 2005;330:82-7.

[67] Brender E. Use of emboli-blocking filters increases, but rigorous data are lacking. JAMA 2006;295:989-90

[68] Owens CA, Bui JT, Knuttinen MG, et al. Pulmonary embolism from upper extremity deep vein thrombosis and the role of superior vena cava filters: a review of the literature. J Vasc Interv Radiol 2010;21:779-87.

[69] Cohen AT, Agnelli G, Anderson FA, et al. VTE Impact Assessment Group in Europe (VITAE). Venous thromboembolism (VTE) in Europe. The number of VTE events and associated morbidity and mortality. Thromb Haemost 2007;98:756-64.

[70] Prandoni P, Lensing AW, Prins $\mathrm{MH}$, et al. Below-knee elastic compression stockings to prevent the post-thrombotic syndrome: a randomized, controlled trial. Ann Intern Med 2004;141:249-56.

[71] Kahn SR, Ginsberg JS. The post-thrombotic syndrome: current knowledge, controversies, and directions for future research. Blood Rev 2002;16:155-65.

[72] Meissner AJ, Misiak A, Ziemski JM, et al. Hazards of thrombolytic therapy in deep vein thrombosis. Br J Surg 1987;74:991-3.

[73] Comerota AJ, Aldridge SC. Thrombolytic therapy for deep venous thrombosis: a clinical review. Can J Surg 1993;36:359-64.

[74] Schweizer J, Kirch W, Koch, et al. Short- and long-term results after thrombolytic treatment of deep venous thrombosis. J Am Coll Cardiol 2000;36:1336-43. 
[75] Meissner MH, Mewissen M. Early outcome after catheter-directed thrombolysis for acute deep venous thrombosis: follow-up of a national multicenter registry. J Vasc Surg 2008;12:123-8.

[76] Mewissen MW, Seabrook GR, Meissner MH, et al. Catheter-directed thrombolysis for lower extremity deep venous thrombosis: report of a national multicenter registry. Radiology 1999;211:39-49.

[77] Bjarnason H, Kruse JR, Asinger DA, et al. Iliofemoral deep venous thrombosis: safety and efficacy outcome during 5 years of catheter-directed thrombolytic therapy. J Vasc Interv Radiol 1997;8:405-18.

[78] Comerota AJ, Aldridge SC, Cohen G, et al. A strategy of aggressive regional therapy for acute iliofemoral venous thrombosis with contemporary venous thrombectomy or catheter-directed thrombolysis. J Vasc Surg 1994;20:244-54.

[79] Comerota AJ, Kagan SA. Catheter-directed thrombolysis for the treatment of acute liofemoral deep venous thrombosis. Phlebology 2000:15:149-55.

[80] Comerota AJ, Throm RC, Mathias SD, et al. Catheter-directed thrombolysis for iliofemoral deep venous thrombosis improves health-related quality of life. J Vasc Surg 2000;32:130-7.

[81] Semba CP, Dake MD. Iliofemoral deep venous thrombosis: aggressive therapy with catheter-directed thrombolysis. Radiology 1994;191:487-94.

[82] Semba CP, Dake MD. Catheter-directed thrombolysis for iliofemoral venous thrombosis. Semin Vasc Surg 1996;9:26-33.

[83] Verhaeghe R, Stockx L, Lacroix H, et al. Catheter-directed lysis of iliofemoral vein thrombosis with use of rt-PA. Eur Radiol 1997;7:996-1001

[84] Baekgaard N, Broholm R, Just S, et al. Long-term results using catheter-directed thrombolysis in 103 lower limbs with acute iliofemoral venous thrombosis. Eur J Vasc Endovasc Surg 2010;39:112-7.

[85] Elsharawy M, Elzayat E. Early results of thrombolysis vs anticoagulation in iliofemoral venous thrombosis: a randomised clinical trial. Eur J Vasc Endovasc Surg $2002 ; 24: 209-14$
[86] Enden T, Kløw NE, Sandvik L, et al, CaVenT study group. Catheter-directed thrombolysis vs. anticoagulant therapy alone in deep vein thrombosis: results of an open randomized, controlled trial reporting on short-term patency. J Thromb Haemost 2009;7:1268-75.

[87] Verstraete M, Miller GAH, Bounameaux $\mathrm{H}$, et al. Intravenous and intrapulmonary recombinant tissue-type plasminogen activator in the treatment of acute massive pulmonary embolism. Circulation 1988;77:353-60.

[88] Kim HS, Patra A, Paxton BE, et al. Adjunctive percutaneous mechanical thrombectomy for lower-extremity deep vein thrombosis: clinical and economic outcomes. J Vasc Interv Radiol 2006;17:1099-104.

[89] Lin PH, Zhou W, Dardik A, et al. Catheter-direct thrombolysis versus pharmacomechanical thrombectomy for treatment of symptomatic lower extremity deep venous thrombosis. Am J Surg 2006;192:782-8.

[90] de Gregorio M, Gimeno M, Alfonso R, et al. Mechanical fragmentation and intrapulmonary fibrinolysis in the treatment of massive pulmonary embolism hemodynamic repercussions. Arch Bronconeumol 2001;37:58-64.

[91] Fava M, Loyola S, Flores P, et al. Mechanical fragmentation and pharmacologic thrombolysis in massive pulmonary embolism. J Vasc Interv Radiol 1997;8:261-6.

[92] Schmitz-Rode T, Janssens U, Duda SH, et al. Massive pulmonary embolism: percutaneous emergency treatment by pigtail rotation catheter. J Am Coll Cardiol 2000;36:375-80

[93] Schmitz-Rode T, Janssens U, Schild HH, et al. Fragmentation of massive pulmonary embolism using a pigtail rotation catheter. Chest 1998;114:1427-36.

[94] Lee L, Kavinsky C], Spies C. Massive pulmonary embolism: review of management strategies with a focus on catheter-based techniques. Expert Rev Cardiovasc Ther 2010;8:863-73.

[95] Kuo WT, Gould MK, Louie JD, et al. Catheter-directed therapy for the treatment of massive pulmonary embolism: systematic review and meta-analysis of modern techniques. Vasc Interv Radiol 2009;20:1431-40. 